\title{
Совершенствование товарной политики предприятия на основе цифровых технологий
}

\author{
Л. Б. Кудрявцева ${ }^{1 凶}$ \\ ${ }^{1}$ Воронежский государственный университет, Университетская пл., 1, \\ 394018, Воронеж, Российская Федерация
}

Для цитирования: Кудрявцева Л. Б. Совершенствование товарной политики предприятия на основе цифровых технологий // Вестник Воронежского государственного университета. Серия: Экономика и управление. 2021. № 1. С. 68-75. DOI 10.17308/econ.2021.1/3321

\begin{abstract}
Предмет. В маркетинговой деятельности предприятий производственного сектора экономики особое место занимает формирование товарной политики. Ее развитие связано с исследованием возможностей и направлений цифровизации процессов разработки товара, управления жизненным циклом продукта и оптимизации товарного ассортимента.

Цель. Анализ возможностей применения цифровых методов и средств в основных направлениях товарной политики, выявление проблем доступности цифровых технологий для компаний различного масштаба хозяйственной деятельности и поиск способов их решения.

Методология. Использован системный подход, общелогические и эмпирические методы. Осуществлялся анализ научно-практических материалов и опыта предприятий.

Выводы. Рыночная ориентация бизнеса предполагает стратегический подход к формированию ассортимента выпускаемой продукции, разработке новых товаров и выведению их на рынок. Применение цифровых технологий на всех этапах формирования товарной политики позволяет повысить конкурентоспособность продукции и реализовать цель маркетинга с меньшими издержками. Развитие товарной политики на основе цифровых технологий сдерживает ряд проблем, связанных с недостаточным уровнем информационных систем, нехваткой квалифицированных специалистов и недоступностью технологий из-за ограниченности ресурсов.
\end{abstract}

Ключевые слова: методы маркетинга, маркетинг нового товара, оптимизация ассортимента, кастомизация.

\section{Введение}

Товарная политика играет ключевую роль в маркетинге производственного предприятия и затрагивает все сферы его деятельности. Обострение конкуренции и повышение насыщенности рынка разнообразными товарами приводит к тому, что практический маркетинг все чаще концентрирует усилия в сфере продвижения, что, однако, не умаляет значения решений по товару, поскольку покупатель потребляет не обещание, а товар. Степень удовлетворенности покупателя в конечном счете зависит от потребительской ценности товара, формирующейся в результате эффективной товарной политики. Маркетинговые решения по товару предопределяют решения в области сегментирования, позиционирования, ценообразования, сбыта и продвижения. В свою очередь выбранная маркетинговая стратегия и соответствующие стратегии сегментирования и позиционирования формируют требования к образу и потребительским свойствам товара. Различные области деятельности предприятия, такие как проектирование товара, организация его производства, логистика, тесно связаны с товарной политикой, подчиняясь ее задачам.

Маркетинг считается сегодня областью деятельности компаний, где наиболее активно внедряются и используются цифровые технологии. Однако практика маркетинга в условиях цифровой трансформации в значительной степени ограничивается коммуникативной и сбытовой функциями. В известной книге «Интернет-маркетинг и digital-стратегии» более 80 \% материала посвящено веб-аналитике (анализ данных о посещаемости сайта и удов-

(C) Кудрявцева Л. Б., 2021

Вестник ВГУ. Серия: Экономика и управление. 2021. № 1. С. 68-75. 
летворенности его посетителей) и продвижению с использованием интернета и цифровых технологий [6].

Конкретизируя направления маркетинговой деятельности, авторы публикаций, связанных с цифровым маркетингом, упоминают поиск в интернете и SEO-оптимизацию, контент-маркетинг, маркетинг в социальных медиа, e-mail-маркетинг, рекламу в интернете и электронных изданиях [6; 9; 12].

Между тем вся деятельность предприятия (научно-техническая, производственная, сбытовая, коммуникационная) должна основываться на знании потребительского спроса и его изменений в перспективе. Маркетинг означает разработку, производство и сбыт того, на что есть потребительский спрос. Важным является также выявление неудовлетворенных запросов покупателей. Информация о потребителях, конкурентах и других факторах макрои микросреды предприятия является основой принятия маркетинговых решений. К сожалению, в производственном секторе экономики информационные системы маркетинга в большинстве своем примитивны и ограничиваются сбором и обработкой информации об имеющихся покупателях, основных конкурентах и рыночных трендах.

Современные информационные технологии, предполагающие обработку больших данных (big data), применяются в основном крупными компаниями банковского сектора, оптовой и розничной торговли, операторами связи. Маркетинговые информационные системы таких предприятий используют инструменты BI (Business Intelligence), технологии хранилищ данных, data mining и OLAP. Технологии data mining используют для решения маркетинговых задач современный математический аппарат, а наиболее гибкой и эффективной технологической платформой для анализа данных и разработки сценариев является архитектура OLAP/MOLAP, позволяющая выполнять моделирование данных, углубляться в детали и делать обобщения, фильтровать, сортировать и перегруппировывать данные во время анализа.

Целью статьи является исследование возможностей применения цифровых методов и средств в основных направлениях товарной политики, выявление проблем доступности цифровых технологий для компаний различного масштаба хозяйственной деятельности и поиск способов их решения.

\section{Материалы и методология исследования}

Систему методов маркетинга часто представляют в терминах 4P: продукт, цена, распределение, продвижение. Первым и ключевым в этой системе является «продукт». Особое значение имеют маркетинговые решения по «продукту» для производственных предприятий. Заметим, что термин «продукт» означает комплекс маркетинговых решений стратегического и оперативного характера и часто трактуется как товарная политика [8]. Управление товарной политикой предполагает планирование и организацию работ в разных областях деятельности предприятия. Прежде всего необходимо отслеживать состояние рынка на всех этапах жизненного цикла товара на предмет его соответствия нуждам и предпочтениям потребителей. Информация о покупателях и конкурентах необходима для выявления неудовлетворенных потребностей, оценки товара по мере его разработки и выведения на рынок, а также для контроля рыночной эффективности существующих товаров. Другая задача маркетинга в отношении товарной политики предусматривает составление технического задания на разработку нового товара. Маркетинговое управление предполагает обеспечение соответствия требований к потребительской ценности товара с его фактическими свойствами. Потребности, в том числе латентные, нужды и предпочтения потребителей находят свое отражение в техническом задании на разработку нового товара. Еще одна важная задача маркетинга в рамках товарной политики - выбор целевых рынков нового товара и его позиционирование. Эти действия зачастую играют решающую роль в рыночном успехе как новых, так и существующих товаров компании. Вопросы позиционирования следует рассматривать на ранних этапах планирования товара ввиду их тесной взаимосвязи с условиями технического задания. Стратегия позиционирования может разрабатываться для отдельного товара или торговой марки, а также охватывать определенный ассортимент товаров.

Разработка нового продукта является функциональной обязанностью не только дизайнеров, конструкторов и технологов. Важная роль в этом процессе принадлежит специалистам по маркетингу. Создание и внедрение на рынок новых товаров связано, как правило, с высокими затратами и значительным риском. Многие инновации при коммерческом освоении, вы- 
ходе на рынок терпят неудачу. Снижению рисков способствует предварительная аналитическая работа по генерации и отбору идей нового товара, эффективность которой зависит от качества маркетинговых исследований. Специалисты признают, что самое большое количество информации используется при изучении и понимании собственного продукта компаний [18; 21]. При существующих онлайн-возможностях проведение количественных и качественных исследований с использованием ресурсов интернета не представляет особых сложностей и не столь трудоемко, как, например, проведение опросов традиционными методами. Сложные и ресурсно-затратные методы сбора первичных данных: объемные опросы, фокус-группы, эксперименты - осуществляются с помощью интернета с меньшими временными и финансовыми издержками. Определенные сервисы big data предоставляют широкие возможности работы с вторичными данными и получение обработанной и структурированной информации в соответствии с целями исследования.

Одним из наиболее надежных методов маркетинговых исследований является совместный (conjoint) анализ, используемый для изучения процесса формирования потребительских предпочтений и моделирования прогнозов по восприятию рынком новых продуктовых предложений [4]. Данный метод основывается на концепции продукта как совокупности свойств или атрибутов и формирует прогнозы о предпочтениях потребителей в реальной ситуации покупки, на основании достаточно хорошо проработанной математической модели и вполне определенной методики эксперимента. Совместный анализ позволяет осуществить выбор оптимальной конфигурации товара и его упаковки, определить значимость атрибутов товаров и услуг и сегментировать рынок на основе выявленных потребительских предпочтений. Проведение совместного анализа связано со сбором и обработкой большого количества информации, что возможно при использовании таких широко известных программных продуктов, как SAS (Statistical Analysis Software), SPSS (Statistical Package for the Social Sciences) и др.

Информация, полученная в результате маркетинговых исследований, необходима для разработки товара. Под термином «разработка товара» понимается не только проектирование изделия и предшествующие этому научно-исследовательские и опытно-кон- структорские работы, но и формирование программы маркетинга.

Проектирование изделий и технологических процессов нужно признать одним из самых значительных факторов конкурентоспособности. Цифровая трансформация способна обеспечить повышение качества и сокращение времени на этапах принятия решений, реализации проектов и выведения на рынок новой продукции. Уже сегодня наиболее востребованными в производственных отраслях являются цифровое проектирование и аддитивные технологии (3D-printing).

Для любого производственного предприятия весьма актуальной остается проблема управления жизненным циклом продукции. Жизненный цикл товара рассматривается маркетологами как период его существования на рынке, начинающийся с момента коммерческого производства и включающий четыре этапа: выведение на рынок, рост, зрелость, спад. Более глубокий подход к проблеме предполагает также существование этапа разработки продукта. Для разработки продукта предприятия давно используют средства автоматизированного проектирования (САПР), управления данными об изделии (PDM), для инженерных расчетов (CAE), автоматического управления производством (CAM). Начиная с 1990-х годов применяются системы управления ресурсами предприятия (ERP), взаимоотношениями с клиентами (CRM), и поставщиками (SCM). Каждая из этих систем содержит большие массивы данных, но из-за того, что они не объединены друг с другом, возникают проблемы неэффективного использования информации. Применение PLM-систем (product lifecycle management) объединяет отдельные участки автоматизации в едином информационном пространстве и образует сквозной конструкторский, технологический и коммерческий цикл, а также обеспечивает взаимодействие автоматизированных систем многих предприятий. PLM-система представляет собой прикладное программное обеспечение для управления жизненным циклом продукции и является основой, интегрирующей информационное пространство, в котором функционируют CAПP, ERP, PDM, SCM, CRM и другие автоматизированные системы [2].

Процесс проектирования изделия обычно разделяют на концептуальное и рабочее проектирование. На этапе концептуального проектирования осуществляется построение пространственных моделей в CAD/CAM-систе- 
мах. Трехмерные модели могут использоваться для представления концептуальных решений, их анализа и последующего отбора. На этапе рабочего проектирования 3D-модели служат основной формой представления геометрической информации об изделии, позволяют производить прототипы и получать чертежно-конструкторскую документацию в удобном для дальнейшей работы типе файла (DXF - для раскроя листа, STP - для станка с ЧПУ, PDF - для печати, JPG - для размещения изображения на сайте или в тексте).

Проектирование изделий для единичного и мелкосерийного производства предприятиями, действующими на рынке В2В, требует тесного взаимодействия с заказчиком, в ходе которого ведется обсуждение и согласование параметров будущего изделия. Директ-маркетинг реализуется не только в виде адресной рассылки информационных материалов по e-mail и коммуникаций с целевыми потребителями на сайте компании-производителя, но и проведения переговоров в формате видеоконференций или с помощью Skype.

Новый продукт создается с учетом профиля целевого сегмента. Процесс сегментирования рынка может быть несложным, когда менеджеры не ставят перед собой задачу максимально точного определения перспективных сегментов либо не существует необходимости поиска новых группировок потребителей с нетривиальными характеристиками. Компании, осуществляющие массовый маркетинг, или «нишевики», имеющие стабильные связи с небольшой группой потребителей, могут ограничиться анализом уже существующих сегментов для уточнения портретов целевых потребителей и адаптацией CRM-систем в соответствии с изменениями в обслуживаемом сегменте (нише). Также возможно применение относительно простого метода группировок по одному-двум признакам сегментирования. Для компаний, реализующих стратегии выхода на новые сегменты, необходим анализ рынка на основе больших объемов постоянно обновляющейся информации. В этой ситуации становятся востребованными аналитические технологии data mining, позволяющие осуществлять классификацию и кластеризацию потребителей по нескольким переменным с помощью нейронных сетей.

Компании, осуществляющие массовое и крупносерийное производство и действующие на высококонкурентном рынке, как правило, используют стратегии дифференцирования товара с целью создания ощутимых отличий от конкурентов и создания конкурентных преимуществ [7; 14]. Аналитические процедуры, необходимые для дифференциации и позиционирования товара, заключаются в анализе конкурентов, установлении значимых для потребителя атрибутов товара и их ранжирования, определения позиций конкурирующих продуктов по важнейшим атрибутам, идентификации потребностей покупателей. Источниками информации для проведения анализа могут служить опросы потенциальных потребителей с помощью традиционных методов и накопленная корпоративная информация. Использование интернет-сервисов при проведении таких исследований позволяет повысить их качество и снизить затраты.

Одним из актуальных способов дифференцирования продукта становится его «кастомизация», т. е. изготовление продукта на основе серийного производства с учетом индивидуальных запросов покупателя. Кастомизация предполагает понимание потребностей и мотивации клиента, определение совокупности свойств и характеристик продукта и четкую фиксацию пожеланий клиента для передачи заказа в производство. Решение этих задач возможно не только на основе личного контакта, но и с помощью оснащения сайта предприятия соответствующими сервисами и использования CRM-систем. Инструментом оформления пожеланий клиента в рабочий проект могут служить пространственные (3D-) модели.

Важной управленческой задачей является формирование оптимальной номенклатуры (ассортимента) выпускаемой продукции. В зависимости от стратегических целей предприятия выбираются критерии оптимальности. Критериями могут служить показатели широты, длины, глубины, согласованности ассортимента. Принятию решений относительно изменения параметров ассортимента предшествует аналитическая работа, требующая привлечения большого массива разнообразных данных: спрос на продукцию предприятия, изменения в покупательских предпочтениях, ассортиментная политика конкурентов, ресурсы предприятия, экономические риски. Анализ ассортимента осуществляется с помощью комплекса методов: матрицы БКГ, АВС- и XYZ- анализ, метод Дибба - Симкина, при этом используются стандартные программные продукты. Задачу оптимизации ассортимента можно решить через построение математической модели, определив перечень факторов, 
оказывающих влияние на структуру ассортимента, а также целевую функцию [4]. Анализ и оптимизация осуществляются с помощью стандартных программных продуктов, сложность состоит в сборе исходных данных, характеризующих факторы внешней среды. Задачи сбора и обработки данных для анализа решаются сервисами big data.

\section{Обсуждение результатов}

Важнейшей задачей маркетинговой деятельности является разработка продукта, обладающего потребительской ценностью. Эффективность товарной политики современного предприятия зависит во многом от степени использования цифровых технологий на всех стадиях создания и рыночной поддержки продуктов. Этап проектирования изделий является сегодня наиболее оснащенным современными цифровыми технологиями. Цифровое проектирование и моделирование успешно применяются крупными предприятиями и рассматриваются специалистами как наиболее востребованное направление повышения конкурентоспособности продукции.

Получение надежного коммерческого результата зависит не только от качества и издержек проектирования, но и от внешней информации, отражающей потребительские запросы. Высокие риски, связанные с созданием и внедрением на рынок новых товаров, могут быть снижены за счет качества первичной и вторичной информации, на основе которой принимаются решения о проектировании продукции. Гипотетически предприятия располагают большими возможностями в изучении требований рынка - от применения недорогих интернет-сервисов и программных продуктов до технологий big data. Однако их практическое применение сдерживается рядом факторов: недооценкой руководством роли маркетинга и маркетинговой информации; недостаточным уровнем «цифровой» компетентности сотрудников, занятых информационным обеспечением и аналитикой; дефицитом ресурсов для приобретения дорогостоящих программных продуктов или оплаты аутсорсинга маркетинговых исследований. Наиболее востребованной является веб-аналитика сайта предприятия, охватывающая в основном область продвижения товара/услуги: анализ эффективности мероприятий по продвижению, посещаемость веб-ресурса, оценка финансовых операций, измерение конверсии. В области товарной политики степень цифровизации процессов и методов получения информации существенно ниже. Исследования потребительских предпочтений, изучение пользовательского опыта, тестирование товара чаще всего проводятся традиционными методами. Например, по данным ЭКОМАР, доля онлайн-опросов в мире в два раза превышает долю личных интервью, в России пока преобладают оффлайн-опросы ${ }^{1}$.

Цифровизация процессов отбора идей новых продуктов и совершенствования существующих, разработки маркетинговой концепции товара, сегментирования рынка и позиционирования товара, оптимизации производственной номенклатуры и ассортимента является пока недостаточно освоенным направлением товарной политики и в перспективе способна обеспечить эффективность «системы предоставления ценности».

\section{Заключение}

Применение цифровых технологий в маркетинге облегчает доступ к необходимой для принятия маркетинговых решений информации, делает более удобным взаимодействие с клиентами. Сегодня предприятия располагают более широкими возможностями для изучения рыночной конъюктуры, потребностей и потребительских запросов. Процесс совершенствования и кастомизации продукта, повышение его качества обеспечиваются с помощью цифрового проектирования и моделирования, увеличивая точность конструкторских решений и сокращая издержки. Но не все предприятия могут сегодня воспользоваться этими преимуществами из-за несовершенства своих информационных систем, дефицита квалифицированных специалистов и высокой стоимости покупных технологий, адаптированных в соответствии со спецификой компаний.

В условиях динамичного развития рынка чрезвычайно важна скорость реакции на изменения потребительских предпочтений. Цифровые технологии, такие как OLAP/MOLAP, SAS, SPSS, значительно расширяют возможности маркетинговых исследований и повышают адекватность данных и точность результатов. Использование PLM-систем, 3D-моделирование и 3D-печать значительно сокращают издержки при проектировании изделий и обеспечивают возможность производства высококачественной продукции.

${ }^{1}$ Отчет McKinsey \&Company. Цифровая Россия : новая реальность. URL: https://www. mckinsey.com/ru/ourinsights 
Применение цифровых технологий при проектировании и производстве новых товаров становится все доступнее для средних и малых компаний с ограниченными ресурсами, поскольку увеличиваются возможности использования бесплатных или недорогих интернет-сервисов и мобильных приложений.

\section{Библиографический список}

1. Акулич М. В. Интернет-маркетинг. М. : Дашков и К, 2016.

2. Браун Д. Как передовые компании осуществляют, используют и поддерживают PLM-интеграцию // Информационно-аналитический PLM- журнал. 2017. № 1 (109). С. 35-47.

3. Васяйчева В. А. Моделирование процесса управления инновационной деятельностью предприятий промышленной сферы // Вестник Воронежского государственного университета. Серия: Экономика и управление. 2020. № 4. С. 74-82. DOI: 10.17308/econ.2020.4/3195

4. Голубкова Е. Н., Сидорчук Р. Р. Маркетинговое управление товаром. М. : Дело и Сервис, 2012.

5. Зотов B. В. Ассортиментная политика фирмы. М. : Эксмо, 2007.

6. Кожушко О., Чуркин И., Агеев А. Интернет-маркетинг и digital-стратегии. Принципы эффективного использования. Новосибирск : РИЦ НГУ, 2015.

7. Котлер Ф., Келлер К. Маркетинг-менеджмент. СПб. : Питер, 2018.

8. Кудрявцева Л. Б. Товарная политика предприятий. Воронеж : Издательский дом ВГУ, 2019.

9. Кулагин В., Сухаревски А., Мефферт Ю.Digital@ Scale : настольная книга по цифровизации бизнеса. М. : Интеллектуальная литература, 2019. 293 с.

10. Ламбен Ж.-Ж., Чумпитас Р., Шулинг И. Менеджмент, ориентированный на рынок. СПб. : Питер, 2017. 720 с.

11. Лифиц И. М., Жукова Ф. А. Товарный менеджмент. М. : Юрайт, 2019. 405 с.

12. Маркова Е. С. Проблемы и перспективы развития цифровой экономики России на глобаль-
Конфликт интересов

Автор декларирует отсутствие явных и потенциальных конфликтов интересов, связанных с публикацией настоящей статьи.

ном рынке // ФЭС: Финансы. Экономика. 2018. № 2. C. 21-27.

13. Мешков А. А. Цифровая среда как экономический фактор интернет-маркетинга // Журнал Маркетинг МВА. Маркетинговое управление предприятием. 2016. Т. 7, вып. 2. С. 75-85.

14. Портер M. Конкурентное преимущество : как достичь высокого результата и обеспечить его устойчивость. М. : Альпина Паблишер, 2020.

15. Семенова Т. В., Зыкова А. В. Управление ассортиментной политикой в современных условиях // Уральский научный вестник. 2018. Т. 1, № 3. C. 029-034.

16. Тамберг В. В., Бадьин А. В. Новая рыночная ниша. От идеи к созданию востребованного продукт. М. : Эксмо, 2008.

17. Федько В. П. Маркетинг в России на рубеже веков : реалии, проблемы, перспективы. М. : ИНФРА-М : Академцентр, 2012.

18. Херф С. Как создать продукт, который полюбят. Опыт успешных менеджеров и дизайнеров. М. : Манн, Иванов и Фербер, 2019.

19. Чибисова Е. И. Экономическая сущность товарной политики и анализ ассортиментного ряда // Альманах мировой науки. 2017. № 3-3 (18). С. 82-87.

20. Щепина И. Н., Бородина А. А. Цифровая экономика как одна из моделей развития постиндустриального общества // Вестник Воронежского государственного университета. Серия: Экономика и управление. 2019. № 2. С. 97-105.

21. Юрашев В. А. Количественные показатели контроля конкурентоспособности торговой марки // Маркетинг. 2016. № 6. С. 55-58.
Кудрявцева Людмила Борисовна, кандидат экономических наук, доцент кафедры экономики, маркетинга и коммерции, Воронежский государственный университет, Воронеж, Российская Федерация

E-mail:mill4@yandex.ru

ORCID ID: 0000-0002-9086-9820
Поступила в редакции 10.01.2021

Подписана в печать 04.02.2021 


\title{
Improving the company's product policy based on digital technologies
}

\section{B. Kudryavtseva ${ }^{1 凶}$}

\author{
${ }^{1}$ Voronezh State University, 1 University sq., 394018, Voronezh, Russian Federation
}

Cite as: Kudryavtseva, L. B. Improving the company's product policy based on digital technologies. Proceedings of the Voronezh State University. Series: Economics and Management. 1, 68-75. (In Russ., abstract in Eng.). DOI: 10.17308/econ.2021.1/3321

\begin{abstract}
Subject. In the marketing activities of enterprises in the manufacturing sector of the economy, a special place is occupied by the formation of a product policy. Its development is associated with the research of the possibilities and directions of digitalization of product development processes, product lifecycle management and product range optimization.

Purpose. Analysis of the possibilities of applying digital methods in the main directions of a product policy, identification of problems of accessibility of digital technologies for companies of various scales of economic activity and search for ways to solve them.

Methodology. A systematic approach, general logical and empirical methods were used. The analysis of scientific and practical materials and experience of enterprises was carried out.

Results. The effectiveness of the product policy of a modern enterprise depends largely on the degree of use of digital technologies at all stages of the creation and market support of products. Their practical application is constrained by a number of factors: management's underestimation of the role of marketing and marketing information; insufficient level of «digital» competence of employees engaged in information support and analytics; lack of resources to purchase expensive software products or pay for outsourcing of marketing research. Today, the most popular is the web analytics of the company's website, which mainly covers the area of product/service promotion. In the field of the product policy, the degree of digitalization of processes and methods of obtaining information is significantly lower.

Digitalization of the processes of selecting ideas for new products and improving existing ones, developing a marketing concept for the product, segmenting the market and positioning the product, optimizing the production nomenclature and assortment is still an insufficiently developed area of product policy and in the future can ensure the effectiveness of the «customer value delivery system».

Conclusions. The market orientation of the business involves a strategic approach to the formation of the product range, the development of new products and their introduction to the market. The use of digital technologies at all stages of the formation of product policy makes it possible to increase the competitiveness of products and realize the goal of marketing with lower costs. The development of a commodity policy based on digital technologies is hindered by a number of problems related to the insufficient level of information systems, the lack of qualified specialists and the unavailability of technologies due to limited resources.
\end{abstract}

Key words: marketing methods, new product marketing, product range optimization, customization.

\section{Conflict of Interest}

The authors declare the absence of obvious and potential conflicts of interest related to the publication of this article.

\section{References}

1. Akulich, M. V. (2016) Internet marketing. Moskow, Dashkov I K publ. (In Russian)

2. Brown, D. (2017) [How leading companies implement, use and support of PLM integration]. Informacionno-analiticheskij PLM-zhurnal. 1 (109). 35-47. (In Russian)
3. Vasyaycheva, V.A. (2020) Modelling the process of industrial enterprises innovative activity managing. Proceedings of Voronezh State University. Series: Economics and Management. 4, 74-82. (In Russian) DOI: 10.17308/econ.2020.4/3195

4. Golubkova, E. N. \& Sydorchuk, R. R. (2012) Marketingovoe upravlenie tovarom [Marketing prod- 
uct management]. Moskow, Delo I Servis publ. (In Russian)

5. Zotov, V. V. (2007) Assortimentnaya politika firmy [Assortment Policy]. Moskow, Eksmo publ. (In Russian)

6. Kozhushko, O. A. \& Churkin, I. \& Ageev, A. (2015) [Internet marketing and digital strategies. Principles of effective use]. Novosibirsk, Novosibirskij gosudarstvennyj universitet publ. (In Russian)

7. Kotler, F. \&. Keller, K. (2018) Marketing menedzhment [Marketing management]. St. Petersburg, Peter publ. (In Russian)

8. Kudryavceva, L. B. (2019) Tovarnaya politika predpriyatij [Product policy of enterprises]. Voronezh, Voronezhskij gosudarstvennyj universitet. (In Russian)

9. Kulagin, V. \& Sukharevsky, A. \& Meffert, Y. (2019) [Digital@Scale]. Nastol'naya kniga po cifrovizacii biznesa. Moscow, Intellectual literature publ.

10. Lamben, Zh.-Zh. (2017) Menedment orientirovannyj na rynok [Managment market-oriented]. St. Petersburg, Peter publ. (In Russian)

11. Lific, I. M. \& Zhukova, I. M. (2019) Tovarnyj menedzhment [Product management]. Moskow, Yurayt publ. (In Russian)

12. Markova, E. S. (2018) Problemy i perspektivy razvitiya cifrovoj ekonomiki Rossii na global'nom rynke [Problems and prospects of development of the digital economy of Russia on the global market]. FES: Finansy. Ekonomika. 2, 21-27. (In Russian)

13. Meshkov, A. A. (2016) Digital environment as an economic factor of Internet marketing. Journal Marketing Management firms. 7 (2), 75-85.

14. Porter, M. (2020) [How to achieve a competitive advantage and ensure its sustainability]. Moscow, Alpina Publisher. (In Russian)
15. Semenova, T. V. \& Zykova, A. V. (2018) Upravlenie assortimentnoj politikoj v sovremennyh usloviyah [Management of assortment policy in modern conditions]. Ural'skij nauchnyj vestnik. 1 (3), 29-34. (In Russian)

16. Tamberg, V. V. \& Bad'in A.V. (2008) Novaya rynochnaya nisha. Ot idei k sozdaniyu vostrebovannogo produkta [New market niche. From the idea to the creation of a popular product]. Moscow, Eksmo publ. (In Russian)

17. Fed'ko, V. P. (2012) Marketing v Rossii na rubezhe vekov: realii, problemy, perspektivy [Marketing in Russia at the turn of the century: realities, problems, prospects]. Moscow, INFRA-M, Akademcentr publ. (In Russian)

18. Herf, S. Kak sozdat' produkt, kotoryj polyubyat. Opyt uspeshnyh menedzherov i dizajnerov [How to create a product that will be loved. Experience of successful managers and designers]. Moscow, Mann, Ivanov and Ferber publ. (In Russian)

19. Chibisova, E. I. (2017) Ekonomicheskaya sushchnost' tovarnoj politiki i analiz assortimentnogo ryada [The economic essence of commodity policy and the analysis of the assortment range]. Al'manah mirovoj nauki. 3-3 (18), 82-87.

20. Shchepina, I. N. \& Borodina, A. A. (2019) Digital economy as one of the models of development of the postindustrial society. Proceedings of Voronezh State University. Series: Economics and Management. 2, 97-105. (In Russian)

21. Yurashev, V. A. (2016) Kolichestvennye pokazateli kontrolya konkurento-sposobnosti torgovoj marki [Quantitative indicators of the control of the competitiveness of a trademark]. Marketing. 6, 55-58. (In Russian)
Ludmila B. Kudryavtseva, Cand. Sci. (Econ.), Assoc. Prof., Economics, Marketing and Commerce Department, Voronezh State University, Voronezh, Russian Federation

E-mail:mill4@yandex.ru

ORCID ID: 0000-0002-9086-9820
Received 10.01.2021

Accepted 04.02.2021 